\title{
Strain gradient plasticity: energetic or dissipative?
}

\author{
N.A. Fleck ${ }^{1}$ and J.R. Willis ${ }^{2}$ \\ University of Cambridge \\ ${ }^{1}$ Department of Engineering, \\ Trumpington Street, Cambridge CB2 1PZ \\ ${ }^{2}$ Department of Applied Mathematics and Theoretical Physics, \\ Wilberforce Road, Cambridge CB3 0WA
}

March 10, 2015

\begin{abstract}
It has been established by computation, and confirmed by analysis, for an infinite slab of strain-gradient sensitive material subjected to plane-strain tensile loading, that passivation of the lateral boundaries at some stage of loading inhibits plastic deformation upon further loading. This result is not surprising in itself except that, remarkably, if the gradient terms contribute to the dissipation, the plastic deformation is switched off completely, and only resumes at a clearly-defined higher load, corresponding to a total strain $\varepsilon_{c}$ say. The analysis presented in this paper confirms the delay of plastic deformation following passivation and determines the exact manner in which the plastic flow resumes. The plastic strain-rate is continuous at the exact point $\varepsilon_{c}$ of resumption of plastic flow and, for the first small increment $\Delta \varepsilon=\varepsilon-\varepsilon_{c}$ in the imposed total strain, the corresponding increment in plastic strain, $\Delta \varepsilon^{p}$, is proportional to $(\Delta \varepsilon)^{2}$. The constant $A$ in the relation $\Delta \varepsilon^{p}(0)=A(\Delta \varepsilon)^{2}$, where $\Delta \varepsilon^{p}(0)$ denotes the plastic strain increment at the centre of the slab, has been determined explicitly; it depends on the hardening modulus of the material. The presence of energetic gradient terms has no effect on the value of $\varepsilon_{c}$ unless the dissipative terms are absent, in which case passivation reduces the rate of plastic deformation but introduces no delay. This qualitative effect of dissipative gradient terms opens the possibility of experimental discrimination of their presence or absence. The
\end{abstract}


analysis employs an incremental variational formulation that is likely to find use in other problems.

Keywords: strain gradient plasticity; boundary passivation; incremental variational principle

\section{Introduction}

Strain gradient plasticity theory is based on the physical notion that geometrically necessary dislocations (GNDs) must exist in crystal plasticity in order to remove the geometric incompatibility associated with lattice curvature and elastic stretch, Nye [1]. The significance of GNDs was recognised by Ashby [2]: GNDs can give additional strengthening by long range elastic back-stress associated with their stored elastic strain energy. Ashby also recognised that short-range interactions occur between GNDs and with statistically stored dislocations (SSDs) to give enhanced hardening. He assumed that the total dislocation density is the sum of the GNDs and SSDs, and they contribute together to provide forest hardening as a dissipative process.

At the local level, SSDs cannot be distinguished from GNDs; rather they are descriptors for mesoscopic measures of the moments of dislocation density, see Groma [3]. The degree to which macroscopic strength depends upon the densities of SSDs and GNDs remains unclear, and the literature continues to combine them in some manner in order to gain an overall effective measure of dislocation density, see for example Evans and Hutchinson [4]. It remains a formidable challenge to model forest hardening as involves the build-up of 3D cells of dislocations, driven by macroscopic plastic strain and plastic strain gradient. An underlying question in strain gradient plasticity theory is the degree to which strain gradients lead to energetic versus dissipative strengthening. This issue arises in the context of phenomenological theories of plasticity (Fleck et al. [5]; Nix and Gao [6]; Gudmundson [7]) in crystal plasticity theory (Gurtin [8] and in discrete dislocation theory van der Giessen and Needleman [9]). Physical arguments have been advanced in order to advocate that energetic or dissipative effects dominate. For example, simple 2D simulations of dislocation arrays on a few slip systems tend to emphasise the role of long range elastic back stress and the associated kinematic hardening, see for example Shu et al [10]. Interfaces can also promote either dissipative or energetic strengthening, depending upon the local slip configuration, as discussed by Fleck and Willis [11]. However, the precise mechanisms by which GNDs lead to material strengthening remain elusive.

A number of specific mechanisms have been proposed for the strengthening associated with GNDs, for example: (i) GNDs combine with SSDs to give forest hardening, leading to dissipative rather than energetic strengthening. This view is supported by some recent experiments by Motz and co-workers $[12,13])$. They measured the effect of beam size upon the cyclic response of copper single crystals under cyclic loading, and observed 
both a pronounced size effect and isotropic hardening. This suggests that the size effect is due to dissipative effects of strain gradients. Likewise, it is generally accepted that the Hall-Petch size effect in polycrystals is not associated with kinematic hardening: the strengthening observed under forward loading is maintained upon cyclic loading. These observations again point towards dissipative strain gradient strengthening. (ii) GNDs arise from the blockage by grain boundaries or by surface passivation, and the associated pile-up of dislocations has a back-stress associated with it, suggestive of energetic strengthening. Likewise, the physical basis for the length scales associated with strain gradients is not fully established, although there have been attempts to derive them, see for example Nix and Gao [6]. Consequently, we remain agnostic as to whether plastic strain gradients lead to energetic and/or dissipative strengthening. The purpose of the present paper is to explore in some detail the sensitivity of the elastic-plastic response of a passivated strip in tension to the presence of dissipative and/or energetic strengthening. A detailed analytical treatment is presented, with a limited set of numerical simulations to illustrate the response immediately post-passivation.

Fleck, Hutchinson and Willis [14] studied the plane-strain stretching of a layer of material occupying the region

$$
\left\{\mathbf{x}:-\infty<x_{1}, x_{3}<\infty,-h<x_{2}<h\right\},
$$

under two different constitutive assumptions. In each case, the material was assumed to be isotropic and incompressible and to conform to a version of gradient plasticity. For one of the materials, the gradient of plastic strain appeared in the free energy - the "energetic" case, while for the other, the gradient of the plastic strain-rate appeared in the dissipation potential - the "dissipative" case ${ }^{1}$. Each material was subjected to a tensile strain $\varepsilon$ in the $x_{1}$-direction, inducing a corresponding contraction $-\varepsilon$ in the $x_{2}$-direction. The surfaces $x_{3}= \pm h$ were initially free of all traction, but were passivated after a specified amount of strain. The energetic material behaved as expected: the plastic strain-rate was reduced after passivation. But in the case of dissipative material, continued plastic strain was completely suppressed, and resumed only after a definite amount of further stretching. This immediately raises two questions: (a) what happens if the boundaries are passivated prior to any straining, and (b) what sort of competition is developed between energetic and dissipative terms, if both are present? Question (a) was addressed in [15] for a solid of finite initial yield strength. Except for a special choice of dissipation potential, gradientdissipative material exhibited a delay in the plastic straining, of the same type as was found in [14], but the relation between the first increment of plastic strain and the corresponding increment in total strain depended on the form of the uniaxial stress-plastic strain relation. It was also found that the gradient-energetic material could display a gap, depending on

\footnotetext{
${ }^{1}$ In [14] and [15], the term "recoverable" was introduced in place of "energetic", and "non-recoverable" was used in place of "dissipative". Here we employ the (so far) more widely used terminology.
} 
the way that the gradient-sensitive modification to the uniaxial stress-plastic strain curve was selected, and restrictions were identified that would avoid it. The present work is devoted to answering question (b). The analysis is "generic" in the sense that, following the uniform plastic straining prior to passivation, the uniaxial stress-plastic strain curve is asymptotically linear, but it is performed for the same form of dissipation potential as was adopted in [14]. It might be expected that other dissipation potentials would induce similar qualitative response though strictly, further analysis would be required.

The plan of the paper is as follows. Section 2 summarizes the governing equations and develops the incremental variational principle that the subsequent analysis employs; this variational principle is likely to be useful also for other applications. Section 3 introduces the plane-strain stretch of a layer, with passivation of the boundaries following an amount of stretch, with uniform plastic strain, under fully traction-free boundary conditions on the sides of the layer. The method of solution is also outlined. The actual solution postpassivation is presented in Section 4. Finally, Section 5 discusses the implications of the findings, their limitations and propects for further work.

\section{Formulation}

\subsection{The governing equations}

Small deformations are assumed. The total strain (denoted $\varepsilon$ ) has components $\varepsilon_{i j}$, the plastic strain $\varepsilon^{p}$ has components $\varepsilon_{i j}^{p}$ and elastic strain $\varepsilon^{e}=\varepsilon-\varepsilon^{p}$. Equivalent plastic strain is $\varepsilon_{P}=\left[(2 / 3) \varepsilon_{i j}^{p} \varepsilon_{i j}^{p}\right]^{1 / 2}$ and equivalent plastic strain-rate is $\dot{e}_{P}=\left[(2 / 3) \dot{\varepsilon}_{i j}^{p} \dot{\varepsilon}_{i j}^{p}\right]^{1 / 2}$. We define also $\varepsilon_{P}^{*}=\left[(2 / 3) \varepsilon_{i j, k}^{p} \varepsilon_{i j, k}^{p}\right]^{1 / 2}$ and $\dot{e}_{P}^{*}=\left[(2 / 3) \dot{\varepsilon}_{i j, k}^{p} \dot{\varepsilon}_{i j, k}^{p}\right]^{1 / 2}$, and further define

$$
\mathcal{E}_{P}=\left(\varepsilon_{P}^{2}+\left(\ell_{E} \varepsilon_{P}^{*}\right)^{2}\right)^{1 / 2}, \quad \dot{E}_{P}=\left(\dot{e}_{P}+\left(\ell_{D} \dot{e}_{P}^{*}\right)^{2}\right)^{1 / 2} .
$$

The medium is assumed to be isotropic and characterized by a free energy function

$$
\psi\left(\varepsilon^{e}, \varepsilon^{p}, \nabla \varepsilon^{p}\right)=\mu \varepsilon_{i j}^{e} \varepsilon_{i j}^{e}+\psi_{P}\left(\varepsilon^{p}, \nabla \varepsilon^{p}\right)
$$

with

$$
\psi_{P}\left(\varepsilon^{p}, \nabla \varepsilon^{p}\right)=F\left(\varepsilon_{P}\right)+G\left(\mathcal{E}_{P}\right) .
$$

The functions $F$ and $G$ are unspecified except that $\Psi_{P}$ is convex, and $F(0)=G(0)=$ $G^{\prime}(0)=0$. The function $\psi_{P}$ so defined is a somewhat generalized version of one employed in [15]. It is not the most general possible but is chosen because it permits the analysis to follow. The length scale $\ell_{E}$ is termed "energetic".

The flow law of the material is defined by the dissipation potential

$$
\varphi\left(\dot{\varepsilon}^{p}, \nabla \dot{\varepsilon}^{p}\right)=\sigma_{0}\left(E_{P}\right) \dot{E}_{P} .
$$


The function $\sigma_{0}$ is unspecified except that it is smooth, and monotone increasing with $\sigma_{0}(0) \geq 0$. The length scale $\ell_{D}$ is termed "dissipative". It is helpful also to define the function

$$
V\left(E_{P}\right)=\int_{0}^{E_{P}} \sigma_{0}(u) d u
$$

such that $\dot{V}=\varphi\left(\dot{\varepsilon}^{p}, \nabla \dot{\varepsilon}^{p}\right)$.

The "energetic" constitutive relations are

$$
\sigma=\frac{\partial \psi}{\partial \varepsilon^{e}}+\bar{\sigma} I, \quad q^{E}=\frac{\partial \psi}{\partial \varepsilon^{p}}, \quad \tau^{E}=\frac{\partial \psi}{\partial \nabla \varepsilon^{p}},
$$

where $\bar{\sigma}=\sigma_{k k} / 3$ and $I$ is the identity tensor. This term is present because the material is assumed to be incompressible. The "dissipative" constitutive relation is

$$
\left(q^{D}, \tau^{D}\right) \in \partial \varphi\left(\dot{\varepsilon}^{p}, \nabla \dot{\varepsilon}^{p}\right)
$$

which implies, under the given assumptions for $\varphi$, that

$$
q_{i j}^{D}=\frac{2}{3} \sigma_{0}\left(E_{P}\right) \frac{\dot{\varepsilon}_{i j}^{p}}{\dot{E}_{P}}, \quad \tau_{i j k}^{D}=\frac{2}{3} \sigma_{0}\left(E_{P}\right) \frac{\ell_{D}^{2} \dot{\varepsilon}_{i j, k}^{p}}{\dot{E}_{P}}
$$

so long as $\dot{E}_{P} \geq 0$, whereas

$$
\Sigma \equiv(3 / 2)^{1 / 2}\left[q_{i j}^{D} q_{i j}^{D}+\tau_{i j k}^{D} \tau_{i j k}^{D} / \ell_{D}^{2}\right]^{1 / 2} \leq \sigma_{0}\left(E_{P}\right)
$$

if $\dot{E}_{P}=0$.

Now with $q=q^{E}+q^{D}$ and $\tau=\tau^{E}+\tau^{D}$, the equilibrium equations are

$$
\operatorname{div} \sigma+f=0, \quad q-\operatorname{div} \tau=s,
$$

where $f$ is body force and $s$ denotes deviatoric stress. Boundary conditions are $\sigma \cdot n=T$, $\tau \cdot n=t^{p}$ if tractions are given, or $u=U, \varepsilon^{p}=\mathcal{E}^{p}$ if kinematic conditions are imposed. Here, $u$ is the displacement vector and $n$ is the outward normal. Mixtures of these conditions, and different combinations of conditions at different boundary points, are allowed.

\subsection{Incremental variational formulation}

For a body occupying a domain $\Omega$, suppose that the fields of displacement $u$ and plastic strain $\varepsilon^{p}$ are known at time $t_{0}$. Then the Cauchy stress $\sigma$ is also known and the plastic strain-rate $\dot{\varepsilon}^{p}$ has to minimize the functional

$$
\Phi_{I}\left(\dot{\varepsilon}^{p}\right)=\int_{\Omega}\left\{\varphi\left(\dot{\varepsilon}^{p}, \nabla \dot{\varepsilon}^{p}\right)-q_{i j}^{0} \dot{\varepsilon}_{i j}^{p}-\tau_{i j k}^{0} \dot{\varepsilon}_{i j, k}^{p}\right\} d x,
$$


where $q^{0}, \tau^{0}$ are any fields that satisfy $q^{0}-\operatorname{div} \tau^{0}=s$, together with any given boundary conditions on the higher-order traction. This is essentially Minimum Principle I of Fleck and Willis $[11,16]$. If the given boundary conditions are homogeneous, the amplitude of the plastic strain-rate is undetermined, and is fixed by a Minimum Principle II which is not recorded here. An approximation for the plastic strain at time $t_{1}=t_{0}+\Delta t$ is then obtained from the forward difference approximation (for any function $f) \dot{f}\left(t_{0}\right) \approx\left(f\left(t_{1}\right)-\right.$ $\left.f\left(t_{0}\right)\right) / \Delta t$. However, this approach fails if $\dot{\varepsilon}^{p}\left(t_{0}\right)=0$, implying that the next increment is determined by an essentially nonlinear equation. Employment of the backward difference formula $\dot{f}\left(t_{1}\right) \approx\left(f\left(t_{1}\right)-f\left(t_{0}\right)\right) / \Delta t$ yields such an equation but (like the forward difference approximation) it has an error of order $\Delta t$. Here, following [6], we opt for a scheme which will deliver a result that is asymptotically exact for the problem of concern. For any continuously-differentiable function $f,\left(f\left(\left(t_{1}\right)-f\left(t_{0}\right)\right) / \Delta t=\dot{f}\left(t_{\gamma}\right)\right.$, where $t_{\gamma}=t_{0}+\gamma \Delta t$ for some $\gamma \in(0,1)$. Thus, if $\varepsilon_{0}^{p}=\varepsilon^{p}\left(t_{0}\right)$ is known, $\varepsilon_{1}^{p}=\varepsilon^{p}\left(t_{1}\right)$ satisfies exactly the field equations and boundary conditions at time $t_{\gamma}$, with $\dot{\varepsilon}^{p}$ expressed as $\left(\varepsilon_{1}^{p}-\varepsilon_{0}^{p}\right) / \Delta t$. This requires the evaluation of $f\left(t_{\gamma}\right)$, which is expressible as $f_{\lambda}=(1-\lambda) f_{0}+\lambda f_{1}$ for some $\lambda \in(0,1)$ if, as is assumed, $\dot{f}\left(t_{\gamma}\right) \neq 0$ and $\Delta t$ is small. In the problem to be considered later, $\gamma$ and $\lambda$ are independent of position in the body and will permit exact study of the asymptotics. More generally, the choice $\gamma=\lambda=1 / 2$ corresponds to employment of the central difference approximation, and has an associated error of order $(\Delta t)^{2}$. The variational principle to follow is expressed in terms general constant $\gamma$ and $\lambda$, leaving the best values open to choice.

The variational statement of the equations governing the first increment is that

$$
\begin{aligned}
\delta\left\{\int _ { \Omega } \left[\psi \left(\varepsilon_{\lambda}^{e}, \varepsilon_{\lambda}^{p}\right.\right.\right. & \left., \nabla \varepsilon_{\lambda}^{p}\right)+V\left(\left(E_{P}\right)_{\lambda}\right)-\sigma_{i j}^{0}\left(\varepsilon_{i j}^{e}\right)_{\lambda} \\
& \left.\left.-q_{i j}^{0}\left(\varepsilon_{i j}^{p}\right)_{\lambda}-\tau_{i j k}^{0}\left(\varepsilon_{i j, k}^{p}\right)_{\lambda}\right]\right\} d x=0,
\end{aligned}
$$

where

$$
\left(E_{P}\right)_{\lambda}=\left(E_{P}\right)_{0}+\lambda \Delta t \dot{E}_{P}
$$

and $\sigma^{0}, q^{0}$ and $\tau^{0}$ satisfy the equilibrium equations and boundary conditions at time $t_{\gamma}$. The variation is taken with respect to $u_{1}$ and $\varepsilon_{1}^{p}$.

\section{The plane-strain tension problem}

When $\Omega$ is the layer (1.1), the system just introduced admits solutions for which the only non-zero components of both total strain and plastic strain are $\varepsilon_{11}\left(x_{2}\right), \varepsilon_{11}^{p}\left(x_{2}\right)$, and $\varepsilon_{22}=$ $-\varepsilon_{11}, \varepsilon_{22}^{p}=-\varepsilon_{11}^{p}$. Elastic incompressibility implies that, if $\sigma_{22}=0$, then $\sigma_{33}=-\sigma_{11} / 2$ so that equilibrium is automatically satisfied. Also, $q_{22}=-q_{11}$ and $\tau_{222}=-\tau_{112}$, all functions 
of $x_{2}$ only, with other components zero. The only remaining requirement is satisfaction of the single higher-order equilibrium equation

$$
q_{11}-\tau_{112,2}=s_{11} \equiv \sigma_{11} / 2 .
$$

The layer (1.1) is to be subjected to a uniform strain $\varepsilon_{11}$, which increases monotonically with time $t$, starting from zero at time $t=0$. The boundaries $x_{2}= \pm h$ are free of all traction up to a time $T$. Plane-strain tension is assumed, so that the only other non-zero component of total strain is $\varepsilon_{22}=-\varepsilon_{11}$. The resulting deformation is elastic up to strain $\sqrt{3} \varepsilon_{Y} / 2$, where $\varepsilon_{Y}$ is the yield strain in simple tension. Thereafter, uniform plastic strain is generated, which is governed by the equilibrium equation (3.1), with the simplification that $\tau_{112}=0$.

Henceforth, suffixes will be dropped so that $\varepsilon_{11}$ becomes $\varepsilon, \varepsilon_{11}^{p}$ becomes $\varepsilon^{p}$ etc. Furthermore, $\varepsilon$ is identified with the time-like variable, the value $\varepsilon_{T}$ corresponding to the clock time $T$. At "time" $\varepsilon_{T}$, the equilibrium equation (3.1) implies that

$$
\sigma_{T} / 2=2 \mu\left(\varepsilon_{T}-\varepsilon_{T}^{p}\right)=q_{T}^{E}+q_{T}^{D},
$$

where

$$
q_{T}^{E}=\frac{1}{\sqrt{3}}\left[F^{\prime}\left(\frac{2 \varepsilon_{T}^{p}}{\sqrt{3}}\right)+G^{\prime}\left(\frac{2 \varepsilon_{T}^{p}}{\sqrt{3}}\right)\right], \quad q_{T}^{D}=\frac{1}{\sqrt{3}} \sigma_{0}\left(\frac{2 \varepsilon_{T}^{p}}{\sqrt{3}}\right) .
$$

Now at time $\varepsilon_{T}$, the boundaries $x_{2}= \pm h$ are passivated, so that $\dot{\varepsilon}^{p}( \pm h)=0$. Plastic flow resumes at time $\varepsilon_{0}$. At this instant, $\varepsilon_{0}^{p}=\varepsilon_{T}^{p}$ but $\varepsilon_{0} \geq \varepsilon_{T}$.

Now consider the next increment of time, up to $\varepsilon_{1}=\varepsilon_{0}+\Delta \varepsilon$. The variational principle (2.12) can be specialized to the assumed form of solution and the resulting functional then made stationary with respect to the unknown $\varepsilon_{1}^{p}$. It generates the equilibrium equation (3.1) at time $\varepsilon_{\gamma}$, with the implication that $\varepsilon_{\lambda}^{e}$ is expressible as

$$
\varepsilon_{\lambda}^{e}=\varepsilon_{\gamma}-\varepsilon_{\lambda}^{p}
$$

Introduce the notation

$$
y=\varepsilon_{1}^{p}-\varepsilon_{0}^{p}, \quad Y=\left(y^{2}+\ell_{D}^{2}\left(y^{\prime}\right)^{2}\right)^{1 / 2},
$$

where $y^{\prime}=d y / d x_{2}$. Since the increment $\Delta \varepsilon$ is small, it suffices to approximate $\psi_{P}$ and $V$ by their three-term Taylor expansions, to give

$$
\psi_{P}\left(\varepsilon_{\lambda}^{p}, \nabla \varepsilon_{\lambda}^{p}\right) \approx \psi_{P}\left(\varepsilon_{0}^{p}, 0\right)+2 q_{T}^{E} \lambda y+2 k_{1} \lambda^{2} y^{2}+2 k_{2} \lambda^{2} \ell_{E}^{2} y^{\prime 2}
$$

where

$$
k_{1}=\frac{1}{3}\left[F^{\prime \prime}\left(\frac{2 \varepsilon_{0}^{p}}{\sqrt{3}}\right)+G^{\prime \prime}\left(\frac{2 \varepsilon_{0}^{p}}{\sqrt{3}}\right)\right], \quad k_{2}=\frac{1}{2 \sqrt{3} \varepsilon_{0}^{p}} G^{\prime}\left(\frac{2 \varepsilon_{0}^{p}}{\sqrt{3}}\right)
$$


and

$$
V\left(\left(E_{P}\right)_{\lambda}\right)=V\left(\frac{2 \varepsilon_{0}^{p}}{\sqrt{3}}\right)+2 q_{T}^{D} \lambda Y+(2 \alpha / 3) \lambda^{2} Y^{2}
$$

where

$$
\alpha=\sigma_{0}^{\prime}\left(\frac{2 \varepsilon_{0}^{p}}{\sqrt{3}}\right) .
$$

The integrand in the variational principle (2.12) can now be given as

$$
\begin{gathered}
f\left(y, y^{\prime}\right)=-2\left[q_{T}^{D}+2 \mu\left(\varepsilon_{0}-\varepsilon_{T}+\gamma \Delta \varepsilon\right)\right] \lambda y+2 \mu \lambda^{2} y^{2}+2 k_{1} \lambda^{2} y^{2} \\
+2 k_{2} l_{E}^{2}\left(y^{\prime}\right)^{2}+2 q_{T}^{D} \lambda Y+\frac{2 \alpha}{3} \lambda^{2} Y^{2}
\end{gathered}
$$

plus a constant. (Equation (3.2) was employed to remove $q_{T}^{E}$ from this expression. Recall also that $\varepsilon_{0}^{p}=\varepsilon_{T}^{p}$.)

Since $\varepsilon_{0}$ and $\Delta \varepsilon$ are independent of $x_{2}$, the Euler-Lagrange equation associated with the variational principle has a first integral, $f-y^{\prime} \partial f / \partial y^{\prime}=c$, constant. Explicitly,

$$
\begin{aligned}
2\left\{-\left[q_{T}^{D}+\right.\right. & \left.2 \mu\left(\varepsilon_{0}-\varepsilon_{T}+\gamma \Delta \varepsilon\right)\right] \lambda y+\mu \lambda^{2} y^{2}+k_{1} \lambda^{2} y^{2} \\
- & \left.\frac{k_{2} \ell_{E}^{2} \lambda^{2}}{\ell_{D}^{2}}\left(Y^{2}-y^{2}\right)+q_{T}^{D} \frac{\lambda y^{2}}{Y}+\frac{\alpha}{3} \lambda^{2}\left(2 y^{2}-Y^{2}\right)\right\}=c .
\end{aligned}
$$

The constant $c$ can be expressed in terms of the value $y_{0}$ of $y$ at $x_{2}=0$ where, by symmetry, $y^{\prime}=0$. Then, with the normalized definitions

$$
\bar{y}=y / y_{0}, \quad \bar{Y}=Y / y_{0},
$$

relation (3.11) implies that

$$
\begin{aligned}
-\left(\frac{\alpha}{3}+\right. & \left.\frac{k_{2} \ell_{E}^{2}}{\ell_{D}^{2}}\right) \lambda y_{0} \bar{Y}^{3}+\left(\left[q_{T}^{D}+2 \mu\left(\varepsilon_{0}-\varepsilon_{T}+\gamma \Delta \varepsilon\right)\right](1-\bar{y})-q_{T}^{D}\right. \\
& \left.+\lambda y_{0}\left[\left(\mu+k_{1}\right)\left(\bar{y}^{2}-1\right)+\frac{\alpha}{3}\left(2 \bar{y}^{2}-1\right)+\frac{k_{2} \ell_{E}^{2}}{\ell_{D}^{2}} \bar{y}^{2}\right]\right) \bar{Y}+q_{T}^{D} \bar{y}^{2}=0 .
\end{aligned}
$$

The solution $y\left(x_{2}\right)$ is now determined by solving (3.13) to give $\bar{Y}$ in terms of $\bar{y}$. Then,

$$
x_{2} / \ell_{D}=\int_{y}^{y_{0}} \frac{d y}{\sqrt{Y^{2}-y^{2}}}=\int_{\bar{y}}^{1} \frac{d \bar{y}}{\sqrt{\bar{Y}^{2}-\bar{y}^{2}}} .
$$

The still-unknown constant $y_{0}$ is fixed by the boundary condition that $y=0$ at $x_{2}=h$ :

$$
h / \ell_{D}=\int_{0}^{1} \frac{d \bar{y}}{\sqrt{\bar{Y}^{2}-\bar{y}^{2}}} .
$$




\section{Properties of the solution}

\subsection{The "generic" case $\ell_{D} / \ell_{E}=O(1)$}

As $\Delta \varepsilon \rightarrow 0$, the amplitude of the increment $y$ of plastic strain tends to 0 . The form of the solution of (3.13) as $y_{0} \rightarrow 0$ is therefore of interest. With the transformation

$$
\bar{Y}=\left(\frac{q_{T}^{D} \bar{y}^{2}}{\left(\alpha / 3+k_{2} \ell_{E}^{2} / \ell_{D}^{2}\right) \lambda y_{0}}\right)^{1 / 3} X
$$

and the definition

$$
R_{\gamma}=\frac{q_{T}^{D}+2 \mu\left(\varepsilon_{0}-\varepsilon_{T}+\gamma \Delta \varepsilon\right)}{q_{T}^{D}},
$$

equation (3.13) can be written

$$
X^{3}-a X-1=0,
$$

where

$$
\begin{aligned}
a= & \left(\frac{q_{T}^{D} \bar{y}^{2}}{\left(\alpha / 3+k_{2} \ell_{E}^{2} / \ell_{D}^{2}\right) \lambda y_{0}}\right)^{1 / 3}\left\{\frac{R_{\gamma}(1-\bar{y})-1}{\bar{y}^{2}}\right. \\
& \left.+\frac{\lambda y_{0}\left[\left(\mu+k_{1}\right)\left(\bar{y}^{2}-1\right)+(\alpha / 3)\left(2 \bar{y}^{2}-1\right)+\left(k_{2} \ell_{E}^{2} / \ell_{D}^{2}\right) \bar{y}^{2}\right]}{q_{T}^{D} \bar{y}^{2}}\right\} .
\end{aligned}
$$

Define

$$
y_{\gamma}^{*}=\left(R_{\gamma}-1\right) / R_{\gamma} .
$$

Then, as $y_{0} \rightarrow 0$,

$$
a \rightarrow \begin{cases}+\infty & \text { if } \bar{y}<y_{\gamma}^{*}-\delta \\ -\infty & \text { if } \bar{y}>y_{\gamma}^{*}+\delta\end{cases}
$$

for any (small, fixed) $\delta>0$. Also, the (unique) positive solution of (4.3) has the properties

$$
X \sim\left\{\begin{array}{l}
a^{1 / 2} \text { if } \bar{y}<y^{*}-\delta, \\
-1 / a \text { if } \bar{y}>y^{*}+\delta .
\end{array}\right.
$$

Correspondingly, as $y_{0} \rightarrow 0$,

$$
\begin{aligned}
\bar{Y} & \sim\left[R_{\gamma}(1-\bar{y})-1\right]^{1 / 2}\left(\frac{q_{T}^{D}}{\left(\alpha / 3+k_{2} \ell_{E}^{2} / \ell_{D}^{2}\right) \lambda y_{0}}\right)^{1 / 2} \text { if } \bar{y}<y_{\gamma}^{*}-\delta, \\
& \sim \frac{\bar{y}^{2}}{1-R_{\gamma}(1-\bar{y})} \text { if } \bar{y}>y_{\gamma}^{*}+\delta .
\end{aligned}
$$


Note first from (3.15) that, necessarily,

$$
h / \ell_{D}>\int_{y_{\gamma}^{*}+\delta}^{1} \frac{d \bar{y}}{\sqrt{\bar{Y}^{2}-\bar{y}^{2}}}
$$

and hence, letting $y_{0} \rightarrow 0$ and then $\delta \rightarrow 0$,

$$
h / \ell_{D} \geq \int_{y_{0}^{*}}^{1} \frac{\left[1-R_{\gamma}(1-\bar{y})\right] d \bar{y}}{\bar{y}\left\{\bar{y}^{2}-\left[1-R_{\gamma}(1-\bar{y})\right]^{2}\right\}^{1 / 2}} .
$$

The variable transformation

$$
\bar{y}=\frac{R_{\gamma}-1}{R_{\gamma}-\cos \theta}
$$

reduces this inequality to the form

$$
h / \ell_{D} \geq \int_{0}^{\pi / 2} \frac{\cos \theta d \theta}{R_{\gamma}-\cos \theta}
$$

from which it easily follows that

$$
h / \ell_{D} \geq \frac{2 R_{\gamma}}{\left(R_{\gamma}^{2}-1\right)^{1 / 2}} \tan ^{-1}\left(\left[\frac{R_{\gamma}+1}{R_{\gamma}-1}\right]^{1 / 2}\right)-\frac{\pi}{2} .
$$

Suppose that plastic flow continued immediately following passivation, so that $R_{0}=R_{T}=$ 1. A contradiction is obtained because the inequality (4.13) has to apply for any $\Delta \varepsilon$, however small. The right side of (4.13) decreases as $R_{\gamma}$ increases. Thus, necessarily, for resumption of plastic flow, $R_{\gamma}>R_{c}$, implying that $R_{0} \geq R_{c}$, where $R_{c}$ is the value of $R_{\gamma}$ for which equality is achieved in (4.13). Reasoning given below shows that plastic flow does occur whenever $R_{\gamma}>R_{c}$, implying that $R_{0}=R_{c}$. It may seems surprising that passivation of the boundaries prevents continued plastic flow, even though the yield criterion, defined by equality in (2.9), has already been reached. It is possible, however, to find fields $\left(q^{D}, \tau^{D}\right)$ that do satisfy $(2.9)$, so long as $R_{0} \leq R_{c}$. This was demonstrated in [5], for the same problem as considered now, except that $\psi_{P}$ was absent, by considering fields

$$
q^{D}=\rho \cos \theta, \quad \tau^{D}=\rho \sin \theta,
$$

with $\theta\left(x_{2}\right)$ chosen so that the equilibrium equation (3.1) was satisfied for all $\varepsilon>\varepsilon_{T}$, up to the value $\varepsilon_{0}$ corresponding to $R_{0}=R_{c}$.

Now take $R_{0}=R_{c}$, so that $R_{\gamma}-R_{c}$ is of order $\Delta \varepsilon$. An informal indication of the asymptotic form of the amplitude $y_{0}$ is obtained by adopting the asymptotic forms (4.7) for $X$, even as $\delta \rightarrow 0$. The right side of (4.10) falls short of $h / \ell_{D}$ by an amount

$$
\left(\frac{h / \ell_{D}+\pi / 2+R_{c}}{R_{c}\left(R_{c}^{2}-1\right)}\right)\left(R_{\gamma}-R_{c}\right)+O\left(R_{\gamma}-R_{c}\right)^{2}
$$


and this must be compensated by the integral from 0 to $y_{0}^{*}$. Estimating this from the asymptotic form $(4.7)_{1}$, it follows that

$$
\frac{2\left(R_{c}-1\right)^{1 / 2}}{R_{c}}\left(\frac{\left(\alpha / 3+k_{2} \ell_{E}^{2} / \ell_{D}^{2}\right) \lambda y_{0}}{q_{T}^{D}}\right)^{1 / 2}=\left(\frac{h / \ell_{D}+\pi / 2+R_{c}}{R_{c}\left(R_{c}^{2}-1\right)}\right)\left(R_{\gamma}-R_{c}\right)+O\left(R_{\gamma}-R_{c}\right)^{2} .
$$

Thus, asymptotically,

$$
\lambda y_{0} \sim\left(\frac{\left(h / \ell_{D}+\pi / 2+R_{c}\right)^{2}}{4\left(R_{c}-1\right)\left(R_{c}^{2}-1\right)^{2}}\right)\left(\frac{q_{T}^{D}}{\alpha / 3+k_{2} \ell_{E}^{2} / \ell_{D}^{2}}\right)\left(R_{\gamma}-R_{c}\right)^{2} .
$$

Note that

$$
R_{\gamma}-R_{c}=\frac{2 \gamma \mu \Delta \varepsilon}{q_{T}^{D}}
$$

The result (4.17) can in fact be shown to be asymptotically exact. Supporting evidence is provided in figure 1. This shows the result of computations based on the full solution of equation (3.13) in combination with (3.15). The parameter values that were employed were $\gamma=\lambda=1 / 2$, together with constitutive parameters chosen arbitrarily to be $\mu=$ $200 / 3, \quad q_{T}^{D}=1, \quad k_{1}=0$ (achievable by taking $F=-G$ ), $k_{2}=1$ and $\alpha=3$. The length $\ell_{D}$ was considered as fixed and $h / \ell_{D}=10$. The resulting value of $R_{c}$ is 1.0327. Results are shown for $\ell_{E} / \ell_{D}$ equal to $1 / 3,1$ and 3 . The predictions of the asymptotic formula (4.17) in combination with (4.18) are also shown. The very small values of $\Delta \varepsilon$ necessary for achievement of the asymptotic result should be noted. These values result in serious loss of significant figures in the computation, which would be even worse if larger $\mu$ or $h / \ell_{D}$ were chosen.

Regardless of the choice of $\gamma$ and $\lambda$, it is predicted that $y_{0} \propto(\Delta \varepsilon)^{2}$. The correct value for the constant is obtained by taking $\gamma=1 / 2$ and $\lambda=1 / 4$. In summary, the presence of $\ell_{E}$ has no effect on the value of $R_{c}$ but it reduces the constant of proportionality between the amplitude $y_{0}$ of the first increment of plastic strain and the square of the corresponding increment $\Delta \varepsilon$ of the imposed total strain.

\subsection{Special case: $\ell_{D}=0$}

When $\ell_{D}=0, Y=y$ and it is easy to confirm that plastic deformation continues immediately following passivation. Assuming that this is so, $\varepsilon_{0}=\varepsilon_{T}$. The limiting form of relation (3.11) as $\ell_{D} \rightarrow 0$ gives

$$
k_{2} \ell_{E}^{2} \lambda^{2} y^{\prime 2}-\left(\mu+k_{1}+\frac{\alpha}{3}\right) \lambda^{2} y^{2}+2 \mu \gamma \Delta y \lambda y=-\left(\mu+k_{1}+\frac{\alpha}{3}\right) \lambda^{2} y_{0}^{2}+2 \mu \gamma \Delta \varepsilon \lambda y_{0} .
$$




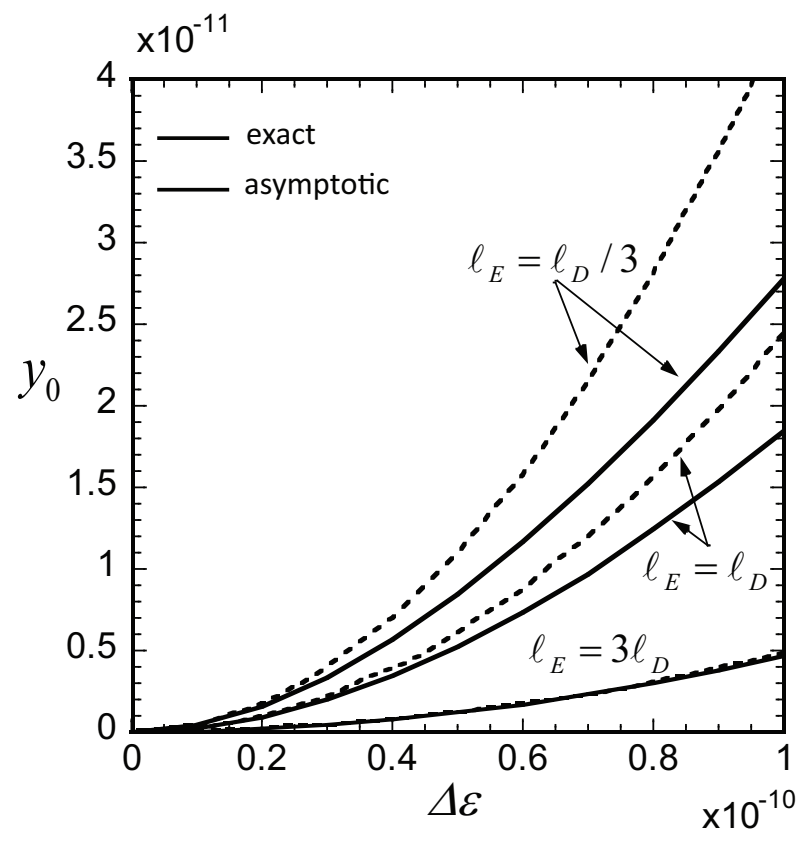

Figure 1: Plots of $y_{0}=\Delta \varepsilon^{p}(0)$ versus $\Delta \varepsilon$ for three values of $\ell_{E} / \ell_{D}$. Solid lines: solution of equation (3.13), dashed lines: asymptotic formula (4.17). 
Equivalently,

$$
\begin{aligned}
k_{2} \ell_{E}^{2} \lambda^{2} y^{\prime 2}-(\mu+ & \left.k_{1}+\frac{\alpha}{3}\right)\left(\lambda y-\frac{2 \mu \gamma \Delta \varepsilon}{\mu+k_{1}+(\alpha / 3)}\right)^{2} \\
& =-\left(\mu+k_{1}+\frac{\alpha}{3}\right)\left(\lambda y_{0}-\frac{2 \mu \gamma \Delta \varepsilon}{\mu+k_{1}+(\alpha / 3)}\right)^{2},
\end{aligned}
$$

whose solution (with $y^{\prime}(0)=0$ ) can be given immediately as

$$
\lambda y=\frac{2 \mu \gamma \Delta \varepsilon}{\left(\mu+k_{1}+\alpha / 3\right)}+\left(\lambda y_{0}-\frac{2 \mu \gamma \Delta \varepsilon}{\left(\mu+k_{1}+\alpha / 3\right)}\right) \cosh \left(\left[\frac{\mu+k_{1}+\alpha / 3}{k_{2}}\right]^{1 / 2} \frac{x_{2}}{\ell_{E}}\right) .
$$

The requirement that $y(h)=0$ now gives

$$
\lambda y_{0}=\frac{2 \mu \gamma \Delta \varepsilon}{\left(\mu+k_{1}+\alpha / 3\right)}\left[1-\operatorname{sech}\left(\left[\frac{\mu+k_{1}+\alpha / 3}{k_{2}}\right]^{1 / 2} \frac{h}{\ell_{E}}\right)\right]
$$

and hence

$$
\lambda y=\frac{2 \mu \gamma \Delta \varepsilon}{\left(\mu+k_{1}+\alpha / 3\right)}\left\{1-\frac{\cosh \left(\left[\frac{\mu+k_{1}+\alpha / 3}{k_{2}}\right]^{1 / 2} \frac{x_{2}}{\ell_{E}}\right)}{\cosh \left(\left[\frac{\mu+k_{1}+\alpha / 3}{k_{2}}\right]^{1 / 2} \frac{h}{\ell_{E}}\right)}\right\} .
$$

Since the predicted relation between $y_{0}$ and $\Delta \varepsilon$ is linear, it becomes exact when the central difference approximation $\gamma=\lambda=1 / 2$ is employed. Note that $y_{0}>0$ so long as $\Delta \varepsilon>0$, consistent with the immediate continuation of plastic deformation.

The result (4.23) can alternatively be derived directly from the rate form of the governing equations. The crucial one of these, which follows from (2.8) only when $\ell_{D}=0$, is

$$
\dot{q}^{D}=\frac{2}{3} \alpha \dot{\varepsilon}^{p} .
$$

A similar calculation was performed in [6], in which this type of formulation was termed "incremental".

Note the contrast between this result and that with $\ell_{D} \neq 0$ : in the present case, there is no elastic gap and the amplitude of plastic strain increment is linearly proportional to the imposed total strain increment $\Delta \varepsilon$, though the constant of proportionality is reduced relative to its value just prior to passivation.

\section{Discussion}

Boundary passivation following uniform straining with boundaries free of all traction introduces a jump in the plastic strain-rate, definitely for the models discussed in this work 
and almost certainly for any model of strain-gradient plasticity. The plastic strain-rate is always reduced; the remarkable property of the dissipative model considered here is that the plastic strain-rate is reduced to zero. Plastic flow does not resume until a distinct higher load (or total strain) is reached. Thus, there is an elastic gap: the increase in total strain from $\varepsilon_{T}$ to $\varepsilon_{c}$ is purely elastic. The analysis demonstrated that the presence of energetic gradient terms has no effect whatever on the size of the elastic gap. It is anticipated, therefore, that it should be possible to decide, from experiments, whether or not plastic strain-gradients contribute to dissipation in any particular material.

It is at least of mathematical interest that the first increment of plastic strain following its resumption is proportional to $(\Delta \varepsilon)^{2}$, demonstrating that the plastic strain-rate is continuous for all strains greater than $\varepsilon_{T}$, including $\varepsilon_{c}$.

The source of the elastic gap is the singularity in the constitutive response which is associated with the fact that the dissipation potential $\varphi$ is proportional to $\dot{E}_{P}$. Although our choice of $\dot{E}_{P}$ is special, it must be recognised that rate-independent response requires $\varphi$ to be homogeneous of degree 1 in the plastic strain-rate and its gradient. Introduction of any amount of rate-dependence (for instance, taking $\varphi$ proportional to $\left(\dot{E}_{P}\right)^{n+1}$ ) permits inelastic deformation to continue immediately following passivation of the boundaries, though if $n$ is small, the inelastic deformation is small until the total strain reaches the $\varepsilon_{c}$ established for the rate-independent case (see, for instance [14], [17]). Any version of strain-gradient plasticity should represent a credible macroscopic approximation to a microscopic model which admits discrete dislocations. It is likely that any discrete dislocation model will display some amount of plastic deformation (dislocation motion) immediately following passivation. The dissipative model considered here offers an idealized response, in which a possibly small plastic deformation is approximated as zero plastic deformation. Whether or not a dissipative strain-gradient model is realistic could therefore also be investigated via appropriate discrete dislocation modelling, along the lines of [18], for instance; it is emphasised, however, that discrete dislocation modelling itself involves physical approximations, so the best test of the prediction of the elastic gap must be one based on experiments.

Part of the reason for the choice of dissipative model was to ensure that it would lead to a problem involving only one independent plastic strain variable. It is, at least, known that computations for torsion of a wire, employing a model that allowed for plastic deformation rather than just strain, have demonstrated the presence of an elastic gap [19]. It is not known for certain that employment of some other strain-rate term such as $\left(\dot{e}_{P}^{\beta}+\left(\ell_{D} \dot{e}_{P}^{*}\right)^{\beta}\right)^{1 / \beta}$ would generate an elastic gap; we speculate that it would, though surely the magnitude of the gap would be modified. The incremental variational formulation introduced in Section 2 and developed in Sections 3 and 4 would find relevance for analytic work based on this last-mentioned modification, and is of likely potential use in a variety of computational problems for strain-gradient plasticity. 


\section{References}

1. Nye, J.F.: Some geometrical relations in dislocated crystals. Acta Metallurgica 1153$162(1953)$.

2. Ashby, M.F.: The deformation of plastically non-homogeneous alloys. Phil. Mag. 21 399-424, (1970) doi: 10.1080/14786437008238426.

3. Groma, I.: Link between the microscopic and mesoscopic length-scale description of the collective behavior of dislocations. Phys. Rev. B 56(10), 5807-5813, (1997) doi: 10.1103/PhysRevB.56.5807.

4. Evans, A.G. and Hutchinson J.W.: A critical assessment of strain gradient plasticity. Acta Materialia 57(5) 1675-1688 (2009) doi:10.1016/j.actamat.2008.12.012.

5. Fleck, N. A., Muller, G.N., Ashby, M.F. and Hutchinson, J.W. Strain gradient plasticity: Theory and experiment. Acta Metallurgica et Materialia 42(2) 475-487, (1994) doi:10.1016/0956-7151(94)90502-9.

6. Nix, W. D. and Gao H.: Indentation size effects in crystalline materials: A law for strain gradient plasticity. J. Mech. Phys. Solids 46(3) 411-425 (1998) doi:10.1016/S00225096(97)00086-0.

7. Gudmundson, P.: A unified treatment of strain gradient plasticity. J. Mech. Phys. Solids 49 1379-1406, (2004) doi:10.1016/j.jmps.2003.11.002.

8. Gurtin M. E.: On the plasticity of single crystals: free energy, microforces, plasticstrain gradients. J. Mech. Phys. Solids 48(5) 989-1036 (2000) doi:10.1016/S00225096(99)00059-9.

9. Van der Giessen, E. and Needleman, A.: Discrete dislocation plasticity: a simple planar model. Modelling Simul. Mater. Sci. Engng. 3, 689-735, (1995) doi:10.1088/0965-0393/3/5/008.

10. Shu, J.Y., Fleck, N.A., van der Giessen, E. and Needleman, A.: Boundary Layers in Constrained Plastic Flow: Comparison of Nonlocal and Discrete Dislocation Plasticity. J. Mech. Phys. Solids 49(6) 1361-1395, (2001) doi:10.1016/S0022-5096(00)000740 .

11. Fleck, N.A. and Willis, J.R.: A mathematical basis for strain gradient plasticity. Part 1: scalar plastic multiplier. J. Mech. Phys. Solids 57 161-177, (2009) DOI:10.1016/j.jmps.2008.09.010.

12. Motz, C., Schoeberl, T. and Pippan, R. (2005) Mechanical properties of micro-sized copper bending beams machined by the focused ion beam technique. Acta Materialia 53, 4269-4279, (2005) DOI:10.1016/j.actamat.2005.05.036. 
13. Kiener, D., Motz, C., Grosinger, W., Weygand, D. and Pippan, R.: Cyclic response of copper single crystal micro-beams. Scripta Materialia 63 500-503 (2010) DOI:10.1016/j.scriptamat.2010.05.014.

14. Fleck, N.A., Hutchinson, J.W. and Willis, J.R.: Strain gradient plasticity under nonproportional loading. Proc. R. Soc. A470, (2014) doi.org/10.1098/rspa.2014.0267.

15. Fleck, N.A., Hutchinson, J.W. and Willis, J.R.: Guidelines for constructing strain gradient plasticity theories. To appear in the ASME J. Applied Mechanics.

16. Fleck, N.A. and Willis, J.R.: A mathematical basis for strain gradient plasticity. Part 2: tensorial plastic multiplier. J. Mech. Phys. Solids 57 1045-1057, (2009) DOI:10.1016/j.jmps.2009.03.007.

17. Niordson, C.N. and Legarth, B.N.: Strain gradient effects in cyclic plasticity, J.Mech. Phys. Solids 58 542-557, (2010) DOI:10.1016/j.jmps.2010.01.007.

18. Bittencourt, E., Needleman, A., Gurtin, M.E. and Van der Giessen, E. : A comparison of nonlocal continuum and discrete dislocation plasticity predictions. J. Mech. Phys. Solids 51 281-310, (2003).

19. Bardella, L. and Panteghini, A.: Modelling the torsion of thin metal wires by distortion gradient plasticity. Submitted for publication. 